\title{
Grenze und Mobilität - ein vielfältiges Forschungsgebiet
}

\author{
Larissa Schindler
}

\begin{abstract}
Denkt man an Grenzen, so denkt man in der Regel nicht an Mobilität, sondern eher an ihre Unterbrechung. Und doch sind Grenzen und Mobilität in vielfältigen Formen verknüpft. Mobilität ist deshalb auf verschiedene Weise ein wichtiges Thema in der Grenzforschung, das sich auf mehrere Bereiche erstreckt. Zwei Bereiche stehen bislang im Vordergrund: die Beweglichkeit von (Territorial-)Grenzen und die sozialen Grenzen von Mobilität. Größere Forschungslücken bestehen dagegen im Bereich der Mobilität an Grenzen, in dem deutlich wird, dass Mobilität auch wegen Grenzen entsteht.
\end{abstract}

\section{Schlagwörter}

Mobilitätschancen, Grenzziehungen, Beweglichkeit, Infrastrukturen, soziale Praktiken

\section{Einleitung}

Seit geraumer Zeit geraten Grenzen in Europa in den Blick der öffentlichen Berichterstattung und alltäglicher Diskussionen. Ein Grund dafür sind die komplexen Dynamiken an und um viele Grenzziehungen, wie etwa die Verstärkung der EU-Außengrenze mit einer gleichzeitigen weitgehenden Relativierung ihrer Binnengrenzen (im Schengen-Raum), die Veränderungen der EU-Außengrenze durch die Aufnahme neuer Mitgliedsstaaten oder die vorübergehend wieder ausgebauten Grenzkontrollen innerhalb des Schengen-Raumes im Rahmen der aktuellen Fluchtbewegungen. Diese Dynamiken beeinflussen die Mobilität (von Menschen, aber auch Dingen, Kapital etc.) beträchtlich. Aus sozial- und kulturwissenschaftlicher Sicht wird damit die Frage nach Grenzen und Mobilität in verschiedenen Aspekten relevant: Wie und in welcher Form sind Grenzen selbst beweglich? Welche Formen von Mobilität bringen Grenzen hervor oder verhindern sie? Wie lässt sich, allgemeiner formuliert, der Zusammenhang zwischen Grenzen und Mobilität analytisch fassen?

Vor diesem Hintergrund beschäftigt sich der vorliegende Beitrag mit der Frage, wie sich aus der Sicht der Grenzforschung eine Perspektive auf Mobilität konzipieren lässt. Er setzt dabei folgende Schwerpunkte: Im ersten Abschnitt werden grundlegende Aspekte der aktuellen Grenz- und Mobilitätsforschung skizziert. An ihrer Schnittlinie entstehen verschiedene Forschungsbereiche, die in den folgenden Abschnitten fokussiert werden. Zunächst werden im zweiten Abschnitt die Beweglichkeit von (Territorial-)Grenzen und die damit entstehenden Dynamiken von und um Grenz(ziehungs)praktiken herausgestellt. In diesem Rahmen klingt der Fokus des dritten Abschnitts bereits durch: die sozialen Grenzen von Mobilität. Wie die Ausführungen zeigen, sind gerade diese beiden Bereiche (Beweglichkeit von Grenzen und soziale Grenzen von Mobilität) relativ gut erforscht. Weniger stark untersucht ist ein dritter Bereich: die Mobilität an Grenzen (vierter Abschnitt), d.h. jene situativen (Mobilitäts-)Praktiken, die an Grenzen stattfinden und sie dadurch auch hervorbringen, aufrechterhalten und in ihrer jeweiligen Spezifik charakterisieren. Der Blick auf solche Praktiken zeigt schließlich, dass Mobilität nicht nur trotz, sondern gerade auch wegen Grenzen stattfindet. 


\section{Grenzforschung und Mobilität}

Die Grenzforschung beschäftigt sich zunächst mit territorialen Grenzen und ihren sozialen Bedingungen und Konsequenzen. Sie bewegt sich damit an einem Schnittpunkt zwischen Geografie und Kultur- und Sozialwissenschaften und leistet für beide Bereiche eine wichtige Erweiterung des Blickwinkels. In diesem Sinne halten Monika Eigmüller und Georg Vobruba in der Einleitung ihres aktuellen Überblickswerks zur Grenzsoziologie fest:

„Staatsgrenzen und grenzüberschreitende Prozesse erschließen sich der Soziologie erst, wenn ihr Gegenstand nicht an der Grenze endet. Soziale Prozesse, die mit Grenzen zu tun haben, ergeben sich vielmehr aus dem Spannungsverhältnis zwischen Grenze als Institution und grenzbezogenem Handeln. [...] Grenzen auf Landkarten werden erst dann auch zu Grenzen in Köpfen, mit all den Wirkungen, die Grenzen innewohnen, wenn ein Staat sie bildet“ (Eigmüller/Vobruba 2016a, S. 4).

Die im ersten Satz dieses Zitats angesprochene Erweiterung der Soziologie bedeutet nicht einfach eine Ausdehnung ihres Gegenstandes. Vielmehr legt der Blick auf territoriale Grenzen auch eine Perspektive nahe, die raumbezogene Konzepte und Analysen beinhaltet. Die Thematik wird so in eine zentrale Entwicklung innerhalb der Sozial- und Kulturwissenschaften eingliedert, die mit dem Begriff des spatial turn (vgl. z.B. Lossau/Lippuner 2004; Döring/Thielmann 2008) gefasst wird. Gerade territoriale Grenzen erlauben in besonderer Weise zu verstehen, wie (politische) Strukturierungen des Raumes zu sozialen Wirklichkeiten werden.

Dabei lässt sich der Gegenstand der Grenze unterschiedlich fassen. Monika Eigmüller (2016, S. 54ff.) unterscheidet drei sozialwissenschaftliche Vorstellungen dazu: (1) Essentialistische Ansätze nehmen territoriale Grenzen als gegeben und interessieren sich für jene Kräfte, die sie hervorbringen. (2) Konstruktivistische Ansätze verstehen Grenzen als soziale und kulturelle Konstrukte, die innerhalb der Begrenzung Stabilität erzeugen. Ein am Raumbegriff Georg Simmels (1908/2016) orientierter Ansatz (3) fokussiert die interaktive Hervorbringung von Grenzen. Als Synthese dieser Ansätze fordert Eigmüller, den „dualen Charakter der Grenze“ ernst zu nehmen:

„Eine angemessene sozialwissenschaftliche Grenztheorie muss den Gegenstand der Grenze zwar als Resultat spezifischer historischer und politischer Prozesse betrachten, zugleich aber auch als Produzentin einer eigenen sozialen Ordnung interpretieren " (Eigmüller 2016, S. 49).

Mit diesem Ansatz wird ein komplexer und gleichzeitig dynamischer Gegenstand skizziert, da es um zwei, sich gegenseitig bedingende Prozesse geht: Die Grenze ist das Ergebnis sozialer Prozesse und bringt diese ihrerseits hervor. Damit rücken Dynamiken der Grenzziehung in den Fokus, die nicht nur die Beweglichkeit von Grenzen fokussieren, sondern auch Dynamiken der mit ihnen verbunden sozialen Ordnungen. Gleichzeitig bilden territoriale Grenzen, ähnlich wie bei vielen anderen Grenzforscher*innen (vgl. Richardson 2013), Eigmüllers Forschungsgegenstand.

Grenzforschung wird jedoch auch mit einem weiteren Begriff von Grenzen betrieben. Werner Schiffauer und Kolleg*innen (2018) konzipieren Grenzen als soziales Phänomen in drei Dimensionen: als territoriale, soziale und temporale Trennungen und Verbindungen. Sie versuchen damit eine Synthese sehr verschiedener Ansätze im Bereich und im Umfeld der wissenschaftlichen Forschung zu Grenzen. Verschiedene Autor*innen (vgl. z.B. Burrell 2008; Gerst/ 
Krämer 2017) entwickeln zudem eine praxeologische Perspektive auf Grenzziehungen, beziehen also systematisch Materialität und Zeitlichkeit in die Analyse ein (vgl. auch Leutloff-Grandits in diesem Band). ${ }^{1}$

Einen im Hinblick auf Mobilität (bzw. Bewegung) besonders ambitionierten Ansatz formuliert Thomas Nail (2016; in diesem Band) in seinem Werk Theory of the Border, in dem er eine „kinopolitical“ Perspektive auf Grenzen konzipiert. Er startet mit dem Befund, dass wir in einer Welt von Grenzen leben (vgl. Nail 2016, S. 2). Gleichzeitig sei Sozialität ständig im Fluß („flow“), weshalb man auch Grenzen nicht als statisches Phänomen untersuchen könne. „If the border is the political ground of our time, the flow is our conceptual starting point " (ebd., S. 25). Grenzen unterbrechen, so sein Ansatz, Mobilität nicht, sondern lenken sie und produzieren damit unterschiedliche Formen von Zirkulation. Zudem sind Grenzen selbst ständig in Bewegung.

Wie in diesem kurzen Überblick bereits durchklingt, werden Grenzen im Rahmen der wissenschaftlichen Grenzforschung zum einen als (historisch) bewegliches Phänomen gedacht, zum anderen findet sich ein Fokus auf Grenzen als Trennungen, der erst kürzlich hinterfragt wird (vgl. z.B. Gerst/Krämer 2017; Cooper/Rumford 2013; Schiffauer et al. 2018). Interessiert man sich für die Frage nach Mobilität und Grenzen, so lohnt deshalb ein Blick in die aktuelle Mobilitätsforschung:

Sie ist stark vom New Mobilities Paradigm (vgl. Sheller/Urry 2006; 2016; Sheller 2014) beeinflusst. Im Vordergrund des Ansatzes steht die Feststellung, dass moderne Gesellschaften von Mobilitäten geprägt sind - man spricht von Mobilitäten im Plural, um die Vielfältigkeit des Phänomens zum Ausdruck zu bringen. Es geht nämlich keineswegs nur um die räumliche Mobilität von Menschen, sondern auch um die Bewegung von Dingen und um mobile und immobile Technologien (in Netzwerken), die Mobilitäten ermöglichen, sowie um die Verbreitung von Bildern und Kommunikation durch unterschiedliche Medien (vgl. Sheller/Urry 2006). Aus historiographischer Perspektive wird festgehalten, dass Mobilität nicht nur in vielerlei Hinsicht zunimmt, sondern moderne Gesellschaften von Beginn an prägt (ebd.).

Arbeiten im Bereich des New Mobilities Paradigm richten sich deshalb gegen eine „Metaphysik der Sesshaftigkeit“ (Malkki 1992, S. 31) in den Kultur- und Sozialwissenschaften, die räumliche Mobilität als zentrales Phänomen moderner Gesellschaften übergehen. Die damit verbundene Dynamisierung der Kultur- und Sozialwissenschaften wird breit angesetzt: Fluide Metaphern für Sozialität (vgl. Vannini 2010, S. 114) sowie ein spezifisches methodisches Instrumentarium, „mobile Methoden“ (vgl. Büscher et al. 2010) werden entwickelt. Diese umfassen etwa verschiedene Formen (teilnehmender) Beobachtungen (Marcus 1995; Büscher et al. 2010, S. 8f) oder shadowing (Czwarniawska 2007; Jirón 2010) sowie videogestützte Beobachtungen (Spinney 2011), aber auch die Analyse von Postkarten (Gillen/Hall 2010), von Tage- und Logbüchern (Jirón 2010; Schindler 2020a; b) sowie von Webseiten, Blogs und Apps (Germann Molz 2010; Stempfhuber/Liegl 2016).

Diese kurze Zusammenfassung macht deutlich, dass Grenzforschung tendenziell Trennungen untersucht, Mobilitätsforschung dagegen fluide Phänomene. An der Schnittlinie dieser beiden Ansätze entsteht deshalb ein fruchtbares Forschungsfeld, das beide Aspekte in den Blick nimmt. Dieses Forschungsfeld umfasst (mindestens) drei Bereiche, mit denen sich die folgenden

$1 \mathrm{Zu}$ den Grundlagen praxistheoretischer Ansätze siehe z.B. Schatzki et al. 2000, Reckwitz 2003, Schmidt 2012. 
Abschnitte beschäftigen: die Beweglichkeit von Grenzen und Grenzziehungspraktiken, die sozialen Grenzen von Mobilität und Mobilität an Grenzen.

Dabei gehe ich konzeptuell von einem weiten Begriff von Grenzen und Grenzziehungen aus, der auch soziale und zeitliche Grenzen umfasst (vgl. z.B. Gerst/Krämer 2017; Schiffauer et al. 2018). Aufgrund ihrer spezifischen Bedeutung in modernen Gesellschaften stehen im Folgenden jedoch Staatsgrenzen und die mit ihnen verbundenen Grenzziehungspraktiken im Vordergrund.

\section{Die Beweglichkeit von (Territorial-)Grenzen und Grenz(ziehungs)praktiken}

Während wir im Alltag territoriale Grenzen meist als fixe Entitäten denken, besteht innerhalb der Grenzforschung Einigkeit über ihre prinzipielle Beweglichkeit (vgl. dazu z.B. Eigmüller 2016, S. 55ff.). Sowohl Politik als auch die Natur verändern Grenzen ständig und auch die mit Grenzen verbundenen Praktiken und Regime sind keineswegs unveränderlich. Die Ambivalenz zwischen Beständigkeit und Bewegung bringen Dominik Gerst et al. (2018, S. 5) prägnant auf den Punkt: „eine der maßgeblichen Paradoxien von Grenzziehungen [ist]: dass sie, obwohl als willkürlicher Akt der Grenzziehung eingesetzt, einen Anspruch auf Eindeutigkeit und Natürlichkeit einfordern“.

Politische Veränderungen von Grenzen sind vor allem aus historischer Perspektive offensichtlich, lassen sich aber auch in der jüngeren Geschichte und in der Gegenwart gut beobachten. Die Entstehung von Nationalstaaten am Beginn der Moderne (vgl. Anderson 1986/2006) brachte die heute geläufige Vorstellung von nationalen Grenzen als Umschließung eines lückenlos regierten Territoriums hervor. Im Zuge der damit verbundenen Nationalisierungsprozesse nahm die Bedeutung dieser Grenzen gegenüber anderen (territorialen) Grenzen (innerhalb der Nationalstaaten) nach und nach zu:

„As a matter of fact, travelers of the late eighteenth century had to undergo harsher controls at city gates and on rivers than at state borders. In many cases, despite colorful political maps already in existence at the time, one would regularly cross state borders and not even notice it“ (Musekamp 2017, S. 3).

Waren also Ende des 18. Jahrhunderts Stadt- und Flussgrenzen für Reisende an vielen Stellen eindrücklicher als Staatsgrenzen, so änderte sich dieses Verhältnis im Laufe des folgenden Jahrhunderts. ${ }^{2}$ Man kann an dieser Stelle einwenden, dass auch heute nationale Grenzen an einigen Stellen kaum bemerkbar sind, etwa innerhalb des Schengen-Raumes. ${ }^{3}$ Wie Henk van Houtum (2016, S. 49) am Beispiel der Grenze zwischen den Niederlanden und Deutschland ausführt, bleiben nationale Grenzen jedoch auch hier bedeutend. Im modernen Denken sind sie - mit der Terminologie Beatrix Haselbergers (2014, S. 510f.) - „thick borders“, die unterschiedliche

2 Die Veränderungen von Grenzen und ihrer Bedeutung seit Ende des 18. Jahrhunderts hängt zudem eng mit der Modernisierung und der Technisierung der Gesellschaft zusammen. Gerade auch die zunehmende Technisierung von Mobilität hatte einen bedeutenden Anteil daran, wie der Kulturhistoriker Jan Musekamp (2017) in einer Studie zum Ausbau der „Ostbahn“ im preußisch/deutsch-polnisch/russischen Grenzgebiet zeigt.

3 Festzuhalten ist jedoch, dass die Reisefreiheit nur für Bürger*innen des Schengen-Raumes gilt und dass auch sie an den Schengen-Innengrenzen bei Bedarf ein gültiges Reisedokument vorweisen müssen. Die erneute Ausweitung der Grenzkontrollen in Zuge der Flüchtlingsströme seit 2015 zeigt zudem, wie schnell hier Grenzkontrollen wieder eingeführt werden können. Interessant war allerdings, wie wenig die verstärkten Grenzkontrollen über die betroffenen Regionen hinaus im allgemeinen Bewusstsein verankert waren. 
ökonomische und soziokulturelle Funktionen haben, auch wenn sie alltagspraktisch (für bestimmte Personengruppen) leicht zu passieren sind.

Gerade nationale Grenzen werden normalerweise „für die Ewigkeit“ gezogen, auch wenn sie nie so lange bestehen (vgl. Houtum 2016, S. 51). Die Zeit zwischen Ende des zweiten Weltkriegs und dem Fall der Berliner Mauer gilt als Phase relativ fixer Staatsgrenzen in Europa (vgl. Schiffauer 2013, S. 109f.) und prägt hier bis heute die alltägliche Wahrnehmung von Staatsgrenzen. Das Entstehen verschiedener neuer Staaten (vor allem in den östlichen Bereichen) Europas nach dem Ende des Eisernen Vorhangs macht ihre Beweglichkeit innerhalb Europas besonders deutlich nachvollziehbar. Am Beispiel Polens zeigt die Politik- und Literaturwissenschaftlerin Steffi Marung (2013) die globalen und regionalen Dynamiken rund um dieserart „wandernde Grenzen“ auf. Sie hält fest:

„Grenzen wurden gezogen, um die Bewegung von Menschen, Sachen, Kapital und Ideen zu kontrollieren. Doch unter den Bedingungen eines globalen Zusammenhanges, dem sich keine Gesellschaft seit ca. der Mitte des 19. Jahrhunderts bei Strafe des Verlustes ihrer Entwicklungsdynamik entziehen konnte, blieben diese Grenzen immer nur auf Zeit nützliche Kontrollinstrumente gegenüber den länderübergreifenden Bewegungen “ (ebd., S. 11f.).

Das nach dem Ende des Eisernen Vorhangs zunehmende Bewegen von Grenzen bringt verschiedene Phänomene der Transnationalisierung hervor, die sich keineswegs allein auf Migrant*innen beschränken, wie Werner Schiffauer (2013) ausführt: „In einer bezeichnenden Weise ist jeder einzelne zum transnational geworden - ohne dass er aktiv einen Schritt über die Grenze machen muss“ (ebd., S. 112, Herv. i. O.). Zudem wechseln Menschen im Zuge von Grenzbewegungen immer wieder ihre territoriale Zugehörigkeit, ohne selbst mobil sein zu müssen (vgl. dazu z.B. Mungiu-Pippidi 2004; Bös/Zimmer 2016). Die Einwohner*innen einiger Gebiete der Bukowina und Transkarpatiens etwa wurden im Verlauf des 20. Jahrhunderts vier verschiedenen Staaten zugeordnet (Mungiu-Pippidi 2004, S. 52). Dabei entstand eine Haltung, die die Politikwissenschaftlerin Alina Mungiu-Pippidi folgendermaßen beschreibt:

„These [...] share the belief that national borders travel faster than people. Their only certainty has been that they would always be in Europe. But now that too has given way. The border of the enlarged EU leaves Bukovina and Transcarpathia out“ (ebd., S. 53).

Dieses Beispiel illustriert die Beweglichkeit von Grenzen mit außergewöhnlicher Deutlichkeit. Für die Frage nach Mobilität und Grenzen sind jedoch gerade auch die Veränderungen von Grenz(ziehungs)praktiken besonders relevant. Thomas Nail (2016, S. 165ff.) zeigt anhand der Grenze zwischen Mexiko und den USA, dass aktuelle Grenzen Hybride aus verschiedenen historischen Grenzziehungsformen sind und auch deshalb in sich beweglich. Zaun, Wall, Zelle und Checkpoint treffen aufeinander und materialisieren eine Grenze, die nicht nur historisch beweglich ist, sondern durch Grenzziehungspraktiken, Grenzüberwindungspraktiken (u.a. Löcher oder Tunnels) und geophysikalische Prozesse ständig in Bewegung ist.

Ein weiteres illustratives Beispiel für Veränderungen von Grenzziehungspraktiken und für ihre Tragweite ist die Geschichte des Reisepasses als materielles Dokument für Grenzübertritte. Der Politikwissenschaftler Mark B. Salter hat sich ausführlich mit der Soziodynamik des Reisepasses beschäftigt (vgl. Salter 2003; 2006; 2015). In verschiedenen Beiträgen stellt er zum einen dar, wie die uns heute geläufige Form des Passes im Verlauf mehrerer Jahrzehnte entstand, zum 
anderen, wie Pässe Teil staatlicher Mobilitätspolitiken werden, die oft als Sicherungs- oder Sicherheitsfragen verhandelt werden.

Noch im 19. Jahrhundert waren Reisepässe in der heutigen Form unbekannt. Stattdessen existierten Briefe lokaler Autoritäten, die für bestimmte Routen Reisefreiheit bestätigten und kursorische Personenbeschreibungen enthielten. ${ }^{4}$ Nur in manchen Fällen waren Fotos beigelegt, die jedoch sehr unterschiedliche Form haben konnten. Auch konnten mehrere Personen (Ehefrau, Dienstboten) mit abgebildet sein. Erst in der Zeit des Ersten Weltkrieges kam es zu deutlich verstärkten staatlichen Grenzkontrollen und zu einer Standardisierung von Pässen, die mit einer Regulierung des Passfotos einherging (vgl. Salter 2015, S. 25). Bis heute aber hängen Mobilität und Grenzziehungspraktiken eng zusammen: Die aktuellen Standards für Pässe werden nicht von staatlichen Organisationen, sondern von der Internationalen Organisation für zivile Luftfahrt (ICAO) festgelegt (vgl. Salter 2004, S. 72.f). Nicht nur aus diesem Grund resümiert Salter: „It is not simply that the passport makes this configuration of global mobility possible, but that the passport is a crucial physical part of the infrastructure that acts " (Salter 2015, S. 18).

Grenzziehungspraktiken sind also mit einer Infrastruktur der Grenze verbunden, die sie gleichzeitig mithervorbringen. In diesem sich ständig verändernden Gefüge werden Ankommende nach verschiedenen Kriterien kategorisiert. Damit klingt ein zweites, gut beforschtes Themengebiet im Bereich Grenze und Mobilität an: die (sozialen) Grenzen von Mobilität.

\section{Die (sozialen) Grenzen von Mobilität}

Nicht nur Grenzen und Grenzziehungspraktiken sind beweglich, sondern es gilt auch umgekehrt: Mobilität ist in verschiedener Weise begrenzt. Mit der sozialen Dimension dieser Begrenzungen setzen sich zahlreiche Studien sowohl im Bereich der Grenzforschung als auch in der Mobilitätsforschung und den Disability Studies ${ }^{5}$ auseinander: Der Geograf und Soziologe Rob Imrie (2000) stellt etwa dar, wie sehr westliche Mobilitätspraktiken, -infrastrukturen und -diskurse auf spezifische Körper, auf mobile bodies, ausgerichtet sind und Menschen mit Behinderung strukturell benachteiligen. Im alltäglichen Leben wird ihre Mobilität durch bauliche Hürden (wie etwa unüberwindbare Stufen oder fehlende Leitsysteme) behindert und durch unbedachte Äußerungen von Mitmenschen erschwert. So sehen sich etwa Blinde besonderen Gefahren ausgesetzt, wenn auch Fahrräder auf Gehwegen unterwegs sind, oder berichten bei ihrer Frage nach einem Preis eines Produkts von nutzlosen Antworten wie zum Beispiel, dass dieser ja am Produkt angeführt sei (vgl. ebd., S. 1646; vgl. auch Saerberg 2006). Die Kommunikationswissenschaftlerin Judith A. Nicholson (2016) zeigt rassistische Einschränkungen von Mobilität in den USA auf. Als Fortsetzung der Forschung über rassistische Kontrollpraktiken der Polizei gegen Schwarze konzentriert sich Nicholson auf den (rassistischen) Einsatz von Schusswaffen im Verkehr, auf „gunscapes“ (ebd., S. 557 ff.). Die Präsenz von Waffen sei so alltäglich, dass sie als zentrales Element von Rassismus im Verkehr übersehen werde (vgl. ebd., S. 556). Sie führt jedoch dazu, dass Schwarze im Verkehr einer besonderen Bedrohung

4 Musekamp (2017, S. 9f.) erwähnt, dass man aufgrund der kursorischen Personenbeschreibung und des fehlenden Fotos zu dieser Zeit leicht mit anderer Menschen Legitimationskarten die Grenze passieren konnte. Zur Geschichte des Passes s. auch Nail (2016, S. 96ff. und 147f.).

5 Ein einführender Überblick zum Ansatz der Disability Studies findet sich z.B. bei Goodley 2016 und bei Waldschmidt/Schneider 2015. 
ausgesetzt sind, wodurch ihre Mobilitätsmöglichkeiten mittelbar und unmittelbar einschränkt werden.

Auch Grenzpraktiken betreffen, wie immer wieder (zumeist kritisch) angemerkt wird, nicht alle Reisenden gleich. Anssi Paasi etwa konstatiert:

„Immigrants, refugees, and displaced people often face borders and the processes of bordering in different ways than do transnational capitalists, highly educated elites or even tourists. This situation forces the researchers to consider not only how they should conceptualize borders but also where contemporary borders and boundary-producing practices are actually ,located““ (Paasi 2013, S. 479).

Indem nicht alle Ankommenden an der Grenze gleichbehandelt werden, werden auch die anschließenden Möglichkeiten für Mobilität in unterschiedlicher Form vergeben. In situ werden Kategorisierungen wie (legal oder illegal) Passierende, (vorübergehend) Bleibende, Ausweichende und Abgewiesene hervorgebracht. ${ }^{6}$ Bleibende sind etwa Menschen, die im Bereich der Grenze arbeiten, aber auch jene, die (zumindest vorübergehend) auf- oder festgehalten werden und erst später wieder mobil werden können (und müssen). Auf abstrakter Ebene sind Grenzen an der Herstellung von nationalen Zugehörigkeiten beteiligt und kategorisieren so auch kollektive Zugehörigkeit (oder Nichtzugehörigkeit) und Mobilitätsmöglichkeiten und -notwendigkeiten (siehe auch Höfler/Klessmann in diesem Band). Diese Kategorisierungen (situativ wie abstrakt) beschränken sich nicht auf Menschen, sondern betreffen ebenso Tiere und Dinge. Auch sie können nicht (umstandslos) über alle Grenzen befördert werden.

Diese Prozesse stehen, wie bereits anklingt, in Zusammenhang mit gesellschaftlichen Makrophänomenen wie Globalisierung und Neoliberalismus. Beide sind Gegenstand zahlreicher Forschungen zu sozialen Grenzen von Mobilität, die das zunehmende Auseinanderklaffen von Mobilitätschancen betonen (vgl. z.B. Weiß 2017). Mit der zunehmenden Delokalisierung von Grenzkontrollen werden nationale Grenzen für globale Unternehmen und Hochqualifizierte zunehmend durchlässiger, während es für Arme immer schwieriger wird, sie zu passieren (vgl. Sassen 2015, S. 297; Nail 2016, S. 30). „Global Cities“ werden dabei, so analysiert die Soziologin und Wirtschaftswissenschaftlerin Saskia Sassen (2015, S. 296), zu Grenzzonen („frontier zones“).

Die Möglichkeiten und Einschränkungen von Mobilität, unter anderem an nationalen Grenzen, sind auch Teil einer umfangreichen Regionalstudie der Soziologin Mimi Sheller (vgl. z.B. 2010; 2013; 2016) über Haiti und die Karibik. Unter anderem beschäftigt sie sich darin mit neoliberalen Umstrukturierungen in karibischen Staaten, wie etwa der „Öffnung“ ehemals öffentlicher Infrastruktur wie Häfen, Flughäfen oder Telekommunikation (vgl. Sheller 2009). Sie zeigt auf, dass diese Umstrukturierungen die Möglichkeiten für internationale Unternehmen und touristische Mobilität erweitern und gleichzeitig den Zugang und die Mobilität von Einheimischen begrenzen:

„Now Caribbean islands, already used as offshore tax havens and free trade zones, are being further disembedded from national territories and repackaged as luxury enclaves that are hyper-connected to global metropolitan transport, media, and data flows-at the very same moment that the region's poorest people are trapped by ever-more powerful border control regimes and enforced immobilities, even after disasters such as Haiti's earthquake“ (Sheller 2010, S. 281).

$6 \mathrm{Zu}$ einer allgemeinen Theorie der Humankategorisierung vgl. Hirschauer 2014. 
Sheller (vgl. z.B. 2010, S. 279; 2016, S. 20) betont, dass Ungleichheit unter anderem entsteht, weil Einzelne durchgeschleust werden und andere an vielen Stellen warten müssen, immer mit dem Risiko abgewiesen zu werden. Auch die Möglichkeit, gut ausgestattete Orte zu erreichen, ist Teil globaler Ungleichheit (vgl. Weiß 2017, S. 297 ff.). In unterschiedlicher Form prägen Mobilitätschancen also die soziale Strukturierung (globaler) Gesellschaften.

Solche Strukturen zeigen sich gerade auch im Zusammenhang mit Umweltkatastrophen, wie Sheller (2013) am Beispiel des Erdbebens in Haiti vom Januar 2010 herausarbeitet. Gerade die Konsequenzen der Klimakatastrophe treffen primär arme Menschen. Besonders deutlich wird in diesem Zusammenhang, wie stark lokale Systeme auf internationalen Vernetzungen beruhen. Gerade diese Vernetzungen machen einen historiographischen Blick unumgänglich:

"If we seek to understand the uneven mobilities [...], we need to trace the historical interplay between various technologies of speed (i.e., from air power to fast-track airport screening), territorial control (i.e., from colonial empires to tourist resorts and private islands), and communication and data sharing (from steamship mail services, to passports, biometrics, and trusted traveler databases)“ (Sheller 2016, S. 19f.).

Diese Form der historiographischen Perspektive zielt, wie man im obigen Zitat erkennen kann, nicht allein auf eine Rekonstruktion der historischen Genese aktueller Praktiken ab, sondern auf eine Analyse verschiedener Vernetzungen. Soziale Ungleichheit, so wird deutlich, erschöpft sich nicht in den klassischen (nach wie vor wichtigen) Themen wie Einkommen, Bildung oder Zugang zum Gesundheitssystem (vgl. auch Schäfer in diesem Band). Vielmehr beruht sie unter anderem auf Mobilitätschancen und Reisegeschwindigkeiten, die gerade auch an verschiedenen Grenzen entschieden werden (vgl. auch Weiß 2017). Das betrifft die Möglichkeiten zum einen, anderenorts (d.h. durch Migration, aber auch Tourismus) eine Verbesserung der eigenen Lebensbedingungen zu erreichen, zum anderen, den Effekten von Katastrophen zu entkommen. Soziale Grenzen schränken sehr grundsätzlich die Möglichkeiten für Mobilität ein, auch Grenzen und Verkehrsmittel differenzieren und klassifizieren. Wie aber geschieht das in situ, d.h. im Rahmen alltäglicher Praktiken an Territorialgrenzen?

\section{Mobilität an Grenzen}

Die Frage nach situativen Praktiken an territorialen Grenzen bringt ein weniger breit beforschtes Thema in den Fokus: die Mobilität an Grenzen, d.h. jene meist sehr kleinräumige Mobilität, die an Grenzen stattfindet. ${ }^{7}$ In diesem Rahmen entstehen die bereits erwähnten Kategorisierungen von Reisenden, aber auch verschiedene, manchmal schillernde Lebenswelten rund um die Grenze. Der Blick auf derartige Phänomene hat zwei Prämissen.

Erstens erfordert er in besonderer Form einen methodologischen Ansatz, der Grenzen nicht (mehr) vom Zentrum aus erforscht, sondern - umgekehrt - soziale Ordnung von der Grenze her denkt:

„Anzustreben ist eine Analyse, die nicht Grenzen als im wahrsten Sinne ,peripheres Phänomen am Rande mitberücksichtigt, sondern analytisch an diesen Grenzen ansetzt, um somit auch sozial-kulturelle Ordnungen als etwas sichtbar zu machen, was sich immer erst über mehr oder minder stabile oder fragile Grenzziehungen zu einem Außen ergibt

7 Wichtige Beiträge zu diesem Phänomen finden sich im Bereich der Forschung zu Grenzländern- und zonen, z.B. Schwell 2008, Bruns 2010, Wagner/Eukowski 2010, Wille 2013. 
und dabei unintendiert mannigfache Zwischenzonen produziert“" (Schiffauer et al. 2018, S. 12).

Zweitens geht damit eine Perspektive einher, die Grenzen eher als Zwischenräume oder -zonen konzipiert denn als Linien.

Beschäftigt man sich in dieser Form mit Mobilität an Grenzen, so zeigen sich sehr verschiedene Phänomene. Sie lassen sich in (mindestens) vier Varianten gruppieren: Erstens entsteht Mobilität in einem sehr basalen Sinn, weil Reisende zur Grenze kommen müssen, um sich konventionellen Grenzkontrollen unterziehen (und sie passieren) zu können. ${ }^{8}$ Hier treffen jedoch nicht nur Territorien aufeinander, sondern auch unterschiedliche Gesetzeslagen und Regularien (vgl. Schmidt 2016, S. 266 ff.), die für die Mobilität an und über Grenzen ausschlaggebend sein können.

Mit dieser Variante von Mobilität an der Grenze beschäftigt sich Kathy Burrell (2008) im Rahmen einer empirischen Studie über die Reisepraktiken polnischer Migrant*innen im Vereinigten Königreich. Anhand von Interviews zeigt sie die Materialität und Diversität internationaler Reisen auf. Nicht nur bei der ersten Einreise nach England, sondern im Rahmen jeder weiteren (Heim-)Reise werden soziokulturelle Dynamiken an verschiedenen Grenzen relevant, die mit der besonderen Materialität dieser Zwischenräume einhergehen: Pässe und Visa operationalisieren politische Entscheidungen auf der Ebene von Personen, unterschiedliche Verkehrsmittel prägen die Reise- und Transportmöglichkeiten, Laptops und andere (oft elektronische) Gegenstände verkürzen nicht nur die Wartezeit, sondern bilden auch einen Teil von Selbstdarstellung und Distinktion, wie an Flughäfen besonders deutlich wird. Dabei werden Fluggäste einerseits über die verschiedenen Kontrollinstanzen des Flughafens beobachtet und kontrolliert, andererseits werden sie selbst in verschiedener Form zu Zuschauer*innen (vgl. dazu auch Adey 2007). Grenzen sind auf diese Weise auch abseits der Kontrollen sehr häufig Orte vielfältiger Tätigkeiten, die spezifische Formen von Mobilität hervorbringen (und andere verhindern).

Zweitens entsteht Mobilität an und um Grenzen auch dann, wenn Reisende davon ausgehen müssen oder in situ erfahren, dass man sie nicht passieren lässt. In solchen Fällen können Routen teilweise so gelegt werden, dass ein Grenzübertritt (gänzlich oder an einem bestimmten Punkt) vermieden wird; aber auch ein erfolgloser Grenzübertrittsversuch produziert Mobilität in verschiedener Form. Auch in dieser Hinsicht bestätigt sich der Befund, dass Mobilität ebenso stattfindet, wenn Grenzübertritte eingeschränkt werden (vgl. dazu z.B. Niemczik-Arambaúa 2010).

Thomas Nail (2016) betont, dass gerade durch das Illegalisieren bestimmter Grenzübertritte eine eigene ökonomische Dynamik entsteht, die in verschiedener Form Mobilität erzeugt. Grenzziehungspraktiken wie Kontrollieren, Festhalten, Einsperren, Zurückschicken erfordern eine eigene Infrastruktur, die Teil eines größeren ökonomischen Zusammenhangs sind: Angestellte, Planungsbüros, Bauwerke, Gefängnisse, Technik und dergleichen sind Teil lokaler Ökonomien, die von der Dynamik durch die Grenze profitieren (vgl. ebd., S. 165 ff.). Gleichzeitig hat diese Dynamik auch eine äußerst brutale Seite: „By forcing migration from Mexico and creating a funnel-shaped fence, the US-Mexico border effectively becomes the world's largest centripetal manhunting apparatus“ (ebd., S. 173). Ist der Begriff des „flow“ also, wie oben dargestellt, Nails Ausgangspunkt, so bringen „junctions“ (Häuser, Städte, aber auch nationale

8 Wie oben erwähnt, finden Grenzkontrollen keineswegs nur an physischen Orten der Grenze statt. 
Grenzen) Schleifenbewegungen („loops“) hervor, durch die der Eindruck vorübergehender Stasis entsteht (ebd, S. 27f.).

Drittens produziert das Aufrechterhalten der bereits erwähnten Infrastruktur der Grenze Mobilität in vielfältiger Form. Unterschiedliche (Berufs-)Gruppen wie etwa Grenzkontrolleur*innen, Reinigungs- und Versorgungspersonal, Zulieferer, Techniker"innen und viele andere müssen sich zur Grenze bewegen, um ihrer Arbeit nachgehen zu können. Auch verschiedene Techniken, Dinge und Materialien werden ständig zur Grenze transportiert und von ihr abtransportiert, um sie in Betrieb zu halten.

Viertens beeinflussen Mobilitäten und ihre Infrastrukturen das Leben an Grenzen, wenn im Umland eine Infrastruktur entsteht, die primär auf die Grenze ausgerichtet ist. In einigen Fällen entstehen entlang von Grenzen ganze Lebenswelten, die unter anderem von Grenzpendler*innen und Wohnmigrant*innen getragen werden (vgl. dazu z.B. Wille 2013; Wille/Roos 2018). Die Politikwissenschaftler Antony Cooper und Chris Rumford (2013, S. 113) betonen in diesem Sinne, dass Grenzen immer auch Gelegenheiten für Kommunikation und Vernetzung bieten.

Der Blick auf Mobilität an Grenzen zeigt also sehr verschiedene Varianten von Mobilität auf, die in einem vielschichtigen Forschungsgebiet zusammenfinden. Es umfasst das relativ geradlinige Passieren der Grenze, das gänzliche oder punktuelle Vermeiden eines Grenzübertritts, erfolglose Grenzübertrittsversuche, das (z.B. berufsbedingte) regelmäßige Anreisen zur Grenze, das Nutzen und Bilden von Infrastrukturen der Grenze und das Leben in Grenzräumen, das auch ein regelmäßiges oder häufiges Passieren der Grenze beinhalten kann. Dabei deutet sich bereits eine weitere Dimension dieses Themas an: Mobilität entsteht auch wegen Grenzen.

An Grenzen treffen, wie bereits erwähnt, nicht nur Territorien aufeinander, sondern (zumeist) auch Räume mit unterschiedlichen sozialen Ordnungen. Dabei manifestieren sich Unterschiede unter anderem in den Rechts-, Wirtschafts- und Gesundheitssystemen sowie in den gängigen Sprachen. Grenzübertritte bringen deshalb nicht nur Schwierigkeiten, sondern auch Möglichkeiten mit sich. Grenzen können, so Bettina Bruns (2010), zu Ressourcen werden. Diese spezifischen Möglichkeiten werden normalerweise durch (Grenz-)Mobilität realisiert. Mobilität entsteht so gerade wegen Grenzen, die entscheidend dazu beitragen, Unterschiede zwischen den jeweiligen sozialen Ordnungen aufrechtzuerhalten.

Besonders eindeutige Fälle solcher Mobilität wegen Grenzen sind Grenzeinkäufe, aber auch illegalisierte Tätigkeiten wie Schmuggel oder Prostitution (vgl. dazu z.B. Schmidt 2016). Ohne Grenze und die an ihnen aufeinandertreffenden Unterschiede in den sozialen Ordnungen würden sie in dieser Form nicht stattfinden (und in manchen Fällen anders bewertet werden). Sehr häufig werden solche Tätigkeiten als Beispiel für die Einschränkung von Mobilität an Grenzen diskutiert, wie etwa in Sanneke Kloppenburgs (2013) empirischer Studie über den Drogenschmuggel von der Karibik in die Niederlande. Ihre detaillierte Rekonstruktion zeigt aber auch an verschiedenen Stellen auf, wie in diesem Rahmen Mobilität erst entsteht. So betont sie, dass die historischen Verbindungen zwischen den beiden Ländern spezifische Konditionen für den Schmuggel hervorbringen. Pointiert formuliert Kloppenburg (2013, S. 56): „While the links between the countries make drug smuggling relatively easy, it is the borders that make it so worthwhile.“ Es sind also die ökonomischen Differenzen zwischen den Ländern sowie die historische Verbindung, die jene illegalisierte Mobilität von Menschen und Drogen hervorbringen. Während im Bereich des Flugverkehrs Maßnahmen zur Unterbindung von Schmuggel 
stark ausgeweitet werden (vgl. dazu z.B. Salter 2004; 2006; Kloppenburg 2013), schreibt er sich in anderen Bereichen in die jeweiligen lokalen Ökonomien geradezu ein. So wird Warenschmuggel im kleineren Ausmaß (petty smuggling) an einigen Landgrenzen als eine Art Einkommensquelle toleriert (vgl. dazu Wiegand 1993; Bruns 2010; Wagner 2010). In manchen Regionen wird die wirtschaftliche Notwendigkeit solcher Formen des Kleinschmuggels selbst von offizieller Seite wahrgenommen, wie Martin Barthel (2010) ausführt.

Weniger augenfällig sind weiträumigere Formen von Grenzmobilität, die aber gleichermaßen die Differenzen zwischen Ländern nutzen, wie Studierendenaustauschprogramme (vgl. z.B. Kuhn 2012) oder Medizintourismus (vgl. z.B. Glinos et al. 2010). Dabei zeigt sich, dass die Kombination mehrerer Differenzen ausschlaggebend sein kann. Im Fall von Abtreibungen oder künstlichen Befruchtungen etwa bringen rechtliche und medizinische Differenzen der jeweiligen Länder Auslandsreisen hervor, die eben auch Grenzübertritte erfordern (und damit die Chance für diese Form der Mobilität ungleich verteilen). Im Bereich der Prostitution an Grenzen wiederum finden sich markante Unterschiede in der öffentlichen Berichterstattung (vgl. Schmidt 2016, S. 263f.).

Auch wenn Grenzen Mobilität in vielfältiger Form einschränken oder auch ablenken, so bringen sie sie gleichzeitig auch in verschiedener Form hervor. Die Analyse von Mobilität an Grenzen kann deshalb in besonderer Form dazu beitragen, die Dynamik an Grenzen und damit auch die Ordnung innerhalb der jeweiligen Grenzen sowie jene in den Zwischenzonen und zwischen den getrennten Fragmenten besser zu verstehen.

\section{Fazit}

Denkt man an Grenzen, so denkt man in der Regel nicht an Mobilität, sondern eher an ihre Unterbrechung. In gewisser Weise besteht darin schließlich die Funktion einer Grenze: Sie trennt Territorien (oder auch Gruppen, Schichten oder Zeiten) voneinander ab. Und doch sind Grenzen und Mobilität in vielfacher Weise verbunden. (Mindestens) drei Forschungsbereiche lassen sich festmachen, in denen diese Verbindungen deutlich werden.

Forschungen zur Beweglichkeit von Grenzen, erstens, konterkarieren in mehrfacher Hinsicht das im Alltag geläufige Bild feststehender, linearer Grenzen: Zum einen verändern sich Grenzziehungen ständig und beeinflussen damit in verschiedener Form das alltägliche Leben in den betroffenen Regionen. Zum anderen ist die Wichtigkeit verschiedener (territorialer) Grenzen historisch und regional ebenso kontingent wie die Form der Grenzziehungspraktiken. Grenzen sind, so zeigt sich, komplex und in vielfältiger Weise bewegt.

Studien zu Grenzen von Mobilität, zweitens, erhöhen die Komplexität der Analyse in der sozialen Dimension: An Grenzen Ankommende (Menschen, Dinge etc.) werden keineswegs gleichbehandelt, sondern kategorisiert. Dabei werden auch die Möglichkeiten zur weiteren Mobilität ungleich verteilt, wovon in vielen Fällen die weiteren Lebenschancen direkt beeinflusst werden. Forschungen in diesem Bereich leisten damit einen wichtigen Beitrag zur Ungleichheitsforschung, der (neben klassischen Themen wie Einkommen, Bildung oder Gesundheit) zunehmend berücksichtigt wird.

Forschungsarbeiten zur Mobilität an der Grenze, drittens, bringen eine situationistische Perspektive in die Grenzforschung ein. Sie hinterfragen die Idee der Grenze als Linie besonders deutlich. Indem sie die alltäglichen Praktiken an und mit Grenzen in den Fokus der Analyse rücken, rekonstruieren sie die vielfältigen Grenzräume und -zonen. Dabei wird deutlich, dass 
Grenzen Mobilität zwar erschweren oder behindern können, dass sie Mobilität in unterschiedlicher Form aber auch immer hervorbringen. In einigen Fällen entsteht Mobilität gerade auch wegen einer Grenze.

An der Schnittlinie von Grenzforschung und Mobilitätsforschung findet sich also ein vielfältiges und fruchtbares Forschungsfeld, in dem verschiedene wissenschaftliche Disziplinen gefragt sind. Gerade der dritte hier angeführte Bereich, die Mobilität an Grenzen, beruht bislang auf punktuellen Forschungsarbeiten und zeigt damit Potenzial für weitere Forschungen. Dabei könnten die einführend erwähnten mobile methods hilfreich sein und weiterentwickelt werden. Schaut man auf Mobilität, so findet man (territoriale, soziale und zeitliche) Grenzen; schaut man auf Grenzen, so findet sich Mobilität (in unterschiedlicher Form).

\section{Weiterführende Literatur}

Bruns, Bettina/Miggelbrink, Judith (Hrsg.) (2011): Subverting Borders: Doing Research on Smuggling and Small-Scale Trade. Wiesbaden: Springer.

Büscher, Monika/Urry, John/Witchger, Katian (Hrsg.) (2010): Mobile Methods. Abington/New York: Routledge.

Richardson, Tim (Hrsg.) (2013): Borders and Mobilities. Special Issue der Zeitschrift „Mobilities“.

Urry, John (2000): Sociology beyond societies: mobilities for the twenty first century. London/New York: Routledge.

Wagner, Matthias/Łukowski, Wojciech (Hrsg.) (2010): Alltag im Grenzland: Schmuggel als ökonomische Strategie im Osten Europas. Wiesbaden: VS Verlag für Sozialwissenschaften.

\section{Literaturverzeichnis}

Adey, Peter (2007): May I have your attention': airport geographies of spectatorship, position, and (im) mobility. In: Environment and Planning D: Society and Space 25, H. 3, S. 515-536.

Anderson, Benedict (1986/2006): Imagined Communities. Reflections on the Origin and Spread of Nationalism. 3., überarb. Aufl., London/Brooklyn: Verso.

Barthel, Martin (2010): Standortfaktor und Ressource - Die Bedeutung der polnisch-ukrainischen Grenze für Przemyśl. In: Wagner, Matthias/Kukowski, Wojciech (Hrsg.): Alltag im Grenzland: Schmuggel als ökonomische Strategie im Osten Europas. Wiesbaden: VS Verlag für Sozialwissenschaften, S. 147-160.

Bös, Matthias/Zimmer, Kerstin (2016): Wenn Grenzen wandern. Zur Dynamik von Grenzverschiebungen im Osten Europas. In: Eigmüller, Monika/Vobruba, Georg (Hrsg.): Grenzsoziologie: die politische Strukturierung des Raumes. 2. Aufl., Wiesbaden: Springer, S. 153-182.

Bruns, Bettina (2010): Grenze als Ressource. Die soziale Organisation von Schmuggel am Rande der Europäischen Union. Wiesbaden: VS Verlag für Sozialwissenschaften.

Burrell, Kathy (2008): Materialising the Border: Spaces of Mobility and Material Culture in Migration from Post-Socialist Poland. In: Mobilities 3, H. 3, S. 353-373.

Büscher, Monika/Urry, John/Witchger, Katian (Hrsg.) (2010): Mobile Methods. Abington/New York: Routledge.

Cooper, Antony/Rumford, Chris (2013): Monumentalising the Border: Bordering Through Connectivity. In: Mobilities 8, H. 1, S. 107-124.

Czarniawska, Barbara (2007): Shadowing, or: Fieldwork on the Move. In: Dies.: Shadowing and other Techniques for Doing Fieldwork in Modern Societies. Copenhagen: Liber, S. 20-58.

Döring, Jörg/Thielmann, Tristan (2008): Spatial turn: das Raumparadigma in den Kultur-und Sozialwissenschaften. Bielefeld: transcript.

Eigmüller, Monika (2016): Der duale Charakter der Grenze. In: Dies./Vobruba, Georg (Hrsg.): Grenzsoziologie: die politische Strukturierung des Raumes. 2. Aufl., Wiesbaden: Springer, S. 49-68.

Eigmüller, Monika/Vobruba, Georg (2016a): Einleitung: Warum eine Soziologie der Grenze? In: Dies. (Hrsg.): Grenzsoziologie: die politische Strukturierung des Raumes. 2. Aufl., Wiesbaden: Springer, S. 16.

Germann Molz, Jennie (2010): Connectivity, collaboration, search. In: Büscher, Monika/Urry, John/Witchger, Katian (Hrsg.): Mobile Methods. Abington/New York: Routledge, S. 88-103.

Gerst, Dominik/Krämer, Hannes (2017): Methodologische Prinzipien einer allgemeinen Grenzsoziologie. In: Lessenich, Stephan (Hrsg.): Geschlossene Gesellschaften. Verhandlungen des 38. Kongresses der Deutschen Gesellschaft für Soziologie in Bamberg 2016. http://publikationen.soziologie.de/index.php/ko ngressband_2016/article/view/485, 01.09.2019. 
Gerst, Dominik/Klessmann, Maria/Krämer, Hannes/Sienknecht, Mitja/Ulrich, Peter (2018): Einleitung: Komplexe Grenzen. Aktuelle Perspektiven der Grenzforschung. In: Berliner Debatte Initial 29, H. 1, S. 3-11.

Gillen, Julia/Hall, Nigel (2010): Any mermaids? Early postcard mobilities. In: Büscher, Monika/Urry, John/Witchger, Katian (Hrsg.): Mobile Methods. Abington/New York: Routledge, S. 20-35.

Glinos, Irene A./Baeten, Rita/Helble, Matthias/Maarse, Hans (2010): A typology of cross-border patient mobility. In: Health \& Place 16, H. 6, S. 1145-1155.

Goodley, Dan (2016): Disability studies: An interdisciplinary introduction. London/New York: Sage.

Haselsberger, Beatrix (2014): Decoding borders. Appreciating border impacts on space and people. In: Planning Theory \& Practice 15, H. 4, S. 505-526.

Hirschauer, Stefan (2014): Un/doing Differences. Die Kontingenz sozialer Zugehörigkeiten. In: Zeitschrift für Soziologie 43, H. 3, S. 170 - 191.

Houtum, Henk van (2016): The Mask of the Border. In: Wastl-Walter, Doris (Hrsg.): The Routledge Research Companion to Border Studies. New York: Routledge, S. 49-61.

Imrie, Rob (2000): Disability and discourses of mobility and movement. In: Environment and Planning A 32, H. 9, S. 1641-1656.

Jirón, Paola (2010): On becoming „la sombra/the shadow“. In: Büscher, Monika/Urry, John/Witchger, Katian (Hrsg.): Mobile Methods. Abington/New York: Routledge, S. 36-53.

Kloppenburg, Sanneke (2013): Mapping the Contours of Mobilities Regimes. Air Travel and Drug Smuggling Between the Caribbean and the Netherlands. In: Mobilities 8, H. 1, S. 52-69.

Kuhn, Theresa (2012): Why educational exchange programmes miss their mark: Cross-border mobility, education and European identity. In: Journal of Common Market Studies 50, H. 6, S. 994-1010.

Lossau, Julia/Lippuner, Roland (2004): Geographie und spatial turn. In: Erdkunde 58, H. 3, S. $201-211$.

Malkki, Liisa (1992): National geographic: The rooting of peoples and the territorialization of national identity among scholars and refugees. In: Cultural anthropology 7, H. 1, S. 24-44.

Marcus, George E. (1995): Ethnography in/of the world system: the emergence of multi-sited ethnography. In: Annual Review of Anthropology 24, S. 95-117.

Marung, Steffi (2013): Die wandernde Grenze. Die EU, Polen und der Wandel politischer Räume 19902010. Göttingen: Vandenhoeck \& Rupprecht.

Mungiu-Pippidi, Alina (2004): Europe Moves Eastward: Beyond the New Borders. In: Journal of Democracy 15, H. 1, S. 48-62.

Musekamp, Jan (2017): Otłoczyn and Alexandrovo: Making and Unmaking of Borders in a Multi ethnic Region. Unveröffentlichtes Manuskript, Workshop Infrastrukturen und Arrangements der Grenze, Europa-Universität Viadrina 29.11.2017.

Nail, Thomas (2016): Theory of the Border. Oxford: Oxford University Press.

Nicholson, Judith A. (2016): Don't shoot! Black mobilities in American gunscapes. In: Mobilities 11, H. 4, S. 553-563.

Niemczik-Arambaúa, Mihaela N. (2010): Erschwerung der Grenzüberschreitung im moldauisch-rumänischen Grenzraum und ihre Folgen für die Grenzraumbevölkerung. In: Wagner, Matthias/Łukowski, Wojciech (Hrsg.): Alltag im Grenzland: Schmuggel als ökonomische Strategie im Osten Europas. Wiesbaden: VS Verlag für Sozialwissenschaften, S. 91-112.

Paasi, Anssi (2013): Borders and Border-Crossings. In: Johnson, Nuala C./Schein, Richard H./Winders, Jamie (Hrsg.): The Wiley-Blackwell Companion to Cultural Geography. Malden: John Wiley \& Sons, S. 478-493.

Reckwitz, Andreas (2003): Grundelemente einer Theorie sozialer Praktiken: Eine sozialtheoretische Perspektive. In: Zeitschrift für Soziologie 32, H. 4, S. 282-301.

Saerberg, Siegfried (2006): „Geradeaus ist einfach immer geradeaus“: Eine lebensweltliche Ethnographie blinder Raumorientierung. Konstanz: UVK.

Salter, Mark B. (2003): Rights of Passage: The Passport in International Relations. Boulder/London: Lynne Rienner Publishers.

Salter, Mark B. (2004): Passports, Mobility, and Security: How smart can the border be? In: International Studies Perspectives 5, H. 5, S. 71-91.

Salter, Mark B. (2006): The Global Visa Regime and the Political Technologies of the International Self: Borders, Bodies, Biopolitics. In: Alternatives: Global, Local, Political 31, H. 2, S. 167-189.

Salter, Mark B. (2015): Passport Photos. In: Ders. (Hrsg.): Making Things International 1: Circuits and Motion. Minneapolis: University of Minnesota Press, S. 18-35.

Sassen, Saskia (2015): Old Borders and New Bordering Capabilities. Cities as Frontier Zones. In: Scienza \& Politica XXVII, H. 53, S. 295-306.

Schatzki, Theodor/Knorr Cetina, Karin/Savigny, Eike von (Hrsg.) (2000): The Practice Turn in Contemporary Theory. London: Routledge. 
Schiffauer, Werner (2013): Grenzen im neuen Europa. In: Beichelt, Timm/Chołuj Bożena/Rowe, Gerard C./ Wagenerm Hans-Jürgen (Hrsg): Europa-Studien. Eine Einführung. 2. Aufl., Wiesbaden: Springer S. 109116.

Schiffauer, Werner/Koch, Jochen/Reckwitz, Andreas/Schoor, Kerstin/Krämer, Hannes (2018): Borders in Motion: Durabilität, Permeabilität, Liminalität. In: Working Paper Series „B/Orders in Motion“ 1. Frankfurt/Oder: Viadrina. https://opus4.kobv.de/opus4-euv/frontdoor/index/index/docId/311, 10.08.2019

Schindler, Larissa (2020a): Logbücher: Zur ethnografischen Erforschung von Mobilität. In: Pfadenhauer, Michaela/Scheibelhofer, Elisabeth (Hrsg.): Interpretative Sozialforschung: Die Entwicklung in Wien. Weinheim: Beltz Juventa, S. 197-210.

Schindler, Larissa (2020b): Logbooks. In: Büscher, Monika/Freudendal-Pedersen, Malene/Kesselring, Sven/ Grauslund Kristensen, Nikolaj (Hrsg.): Handbook of Methods and Applications for Mobilities Research. Cheltenham: Edward Elgar Publishing, S. 102-110.

Schmidt, Daniel (2016): „It's not an entertainment.“ Prostitution an Grenzen. In: Eigmüller, Monika/Vobruba, Georg (Hrsg.): Grenzsoziologie: die politische Strukturierung des Raumes. 2. Aufl., Wiesbaden: Springer-Verlag, S. 263-269.

Schmidt, Robert (2012): Soziologie der Praktiken: Konzeptionelle Studien und empirische Analysen. Frankfurt/M.: Suhrkamp.

Schwell, Alexandra (2008): Europa an der Oder: Die Konstruktion europäischer Sicherheit an der deutschpolnischen Grenze. Bielefeld: transcript.

Sheller, Mimi (2009): The new Caribbean complexity: Mobility systems, tourism and spatial rescaling. In: Singapore Journal of Tropical Geography 30, H. 2, S. 189-203.

Sheller, Mimi (2010): Air mobilities on the US-Caribbean border: Open skies and closed gates. In: The Communication Review 13, H. 4, S. 269-288.

Sheller, Mimi (2013): The islanding effect: post-disaster mobility systems and humanitarian logistics in Haiti. In: Cultural Geographies 20, H. 2, S. 185-204.

Sheller, Mimi (2014): The new mobilities paradigm for a live sociology. In: Current sociology 62, H. 6, S. 789-811.

Sheller, Mimi (2016): Uneven Mobility Futures: A Foucauldian Approach. In: Mobilities 11, H. 1, S. 1531.

Sheller, Mimi/Urry, John (2006): The New Mobilities Paradigm. In: Environment and Planning A 38, H. 2, S. 207-226.

Sheller, Mimi/Urry, John (2016): Mobilizing the new mobilities paradigm. In: Applied Mobilities 1, H. 1, S. $10-25$.

Simmel, Georg (1908/2016): Der Raum und die räumliche Ordnung der Gesellschaft. In: Eigmüller, Monika/Vobruba, Georg (Hrsg.): Grenzsoziologie: Die politische Strukturierung des Raumes. Wiesbaden: Springer, S. 9-18.

Spinney, Justin (2011): A Chance to Catch a Breath: Using Mobile Video Ethnography in Cycling Research. In: Mobilities 6, H. 2, S. 161-182.

Stempfhuber, Martin/Liegl, Michal (2016): Intimacy Mobilized: Hook-Up Practices in the Location-Based Social Network Grindr. In: Österreichische Zeitschrift für Soziologie, 41, H. 1, S. 51-70.

Vannini, Phillip (2010): Mobile Cultures: From the Sociology of Transportation to the Study of Mobilities. In: Sociology Compass 4, H. 2, S. 111-121.

Wagner, Matthias (2010): Die moralische Ökonomie des Schmuggels. In: Ders./Łukowski, Wojciech (Hrsg.): Alltag im Grenzland: Schmuggel als ökonomische Strategie im Osten Europas. Wiesbaden: VS Verlag für Sozialwissenschaften, S. 73-90.

Waldschmidt, Anne/Schneider, Werner (Hrsg.) (2015): Disability studies, Kultursoziologie und Soziologie der Behinderung: Erkundungen in einem neuen Forschungsfeld. Bielefeld: transcript.

Weiß, Anja (2017): Soziologie globaler Ungleichheiten. Frankfurt/M.: Suhrkamp.

Wiegand, Bruce (1993): Petty Smuggling as „Social Justice“: Research Findings from the Belize-Mexico Border. In: Social and Economic Studies 42, H. 1, S. 171-193.

Wille, Christian (2013): Zur Persistenz und Informalität von Räumen der Grenze. Theoretisch-konzeptionelle Überlegungen und empirische Befunde. In: Itinera-Schweizerische Zeitschrift für Geschichte 34, S. 99-112.

Wille, Christian/Roos, Ursula (2018): Grenzüberschreitende Lebenswelten an der luxemburgischen Grenze? Eine empirische Annäherung am Beispiel von Grenzpendlern und Wohnmigranten. In: Pallagst, Karina/Hartz, Andrea/Caesar, Beate (Hrsg.): Border Futures-Zukunft Grenze-Avenir Frontière. Zukunftsfähigkeit grenzüberschreitender Zusammenarbeit. Arbeitsberichte der ARL, 20, S. 168-189. https://shop .arl-net.de/border-futures-zukunft-grenze-avenir-frontiere.html, 10.08.2019. 